\title{
Influence of lateral heat diffusion on the thermal impedance measurement of photovoltaic panels
}

\author{
G. De Mey, J. Wyrzutowicz ${ }^{1}$, A. De Vos \\ Department of Electronics and Information Systems, \\ Ghent University, \\ Sint Pietersnieuwstraat 41, 9000 Ghent, Belgium \\ W. Marańda *, A. Napieralski* \\ Deparment of Microelectronics and Computer Science, \\ Lodz University of Technology \\ ul. Wólczańska 221-223, 90-924 Eódź, Poland
}

\begin{abstract}
The thermal time constant distribution of a photovoltaic panel has been measured experimentally. A wide spectrum ranging between a few $m s$ and a few hours have been detected. A simple thermal analysis can only explain time constants up to $500 \mathrm{~s}$. It was found that the lateral heat diffusion inside the solar cells was responsible to the observed phenomena. The reason was the top finger shaped contact and the non uniform characteristics of the individual solar cells give rise to a non uniform power distribution and hence a lateral transient heat transfer.
\end{abstract}

Keywords: thermal impedance, photovoltaic panel, heat conduction, semiconductor thermal measurement

\section{Introduction}

If solar panels are used in northern countries the solar energy is often fluctuating, as can be found from meteorological data [1]. As a consequence,

\footnotetext{
${ }^{*}$ Corresponding author

Email address: maranda@dmcs.p.lodz.pl (W. Marańda )

${ }^{1} \mathrm{~J}$. Wyrzutowicz wants to thank the EU for the financial support within the framework of the Erasmus Socrates student exchange program.
} 
the electricity production will be fluctuating as well, so that only grid coupled systems are appropriate [2, 3]. If stand alone photovoltaic plants are used, very large battery storage is necessary to provide a constant supply during the whole year $[4,5]$.

A fluctuating solar energy input gives also rise to a fluctuating temperature of the solar cells. The efficiency of a solar cell being temperature dependent, one needs an adequate thermal model for a solar panel in order to calculate the overall electricity production from meteorological data. The influence of the temperature on the efficiency is usually neglected.

If one subtracts the delivered electric power from the absorbed solar power, one gets the amount of power $P$ converted into heat. From the thermal resistance $R_{t h}$ of the solar panel, the temperature rise of the solar cells above ambient is then simply given by $R_{t h} P$. However this approach assumes that the solar cells always reach their steady state temperature. As will be shown further on in this paper, the thermal time constant of a solar panel can be as high as $500 \mathrm{~s}$, or almost one hour $(\approx 5$ time constants $)$ is needed to reach steady state. Taking into account that the solar power can vary quite considerably in such a period, it is clear that a thermal analysis should be time dependent. Hence, a thermal resistance has to be replaced by a thermal impedance, which includes the dynamic behavior as well.

One may argue that a day with a fluctuating sunshine will produce less energy as compared to a day with a bright full sunshine. However, in northern countries, the total amount of energy produced over a whole year during all these "fluctuating" days is no longer negligible. Moreover, the electronic inverter system connecting the solar plant with the electricity grid usually has a maximum power much less than the solar peak power. A typical value is a solar panel of $5 \mathrm{~kW}$ peak power being connected to the grid with an inverted system of just $3.6 \mathrm{~kW}$. The reason is quite obvious: the power loss is negligible because there is only a limited period the sunshine is high enough to produce more that $3.6 \mathrm{~kW}$.

In this paper the thermal impedance of a solar panel will be measured. The same technique used for semiconductor components will be applied. It will be shown then that the lateral heat diffusion (= parallel to the panel's surface) makes these measurements more difficult to carry out. Although the thickness of the panel is much smaller than the lateral dimensions, a one dimensional analysis (= perpendicular to the panel's surface) turns out to be inadequate if electric heating is applied during the measurements, although this approach seems to be the most obvious way. 


\section{Simplified thermal analysis}

The solar panels which have been investigated experimentally contain polycrystaline silicon cells fitted between two glasses each having a thickness of $2 \mathrm{~mm}$ (Fig. 1).

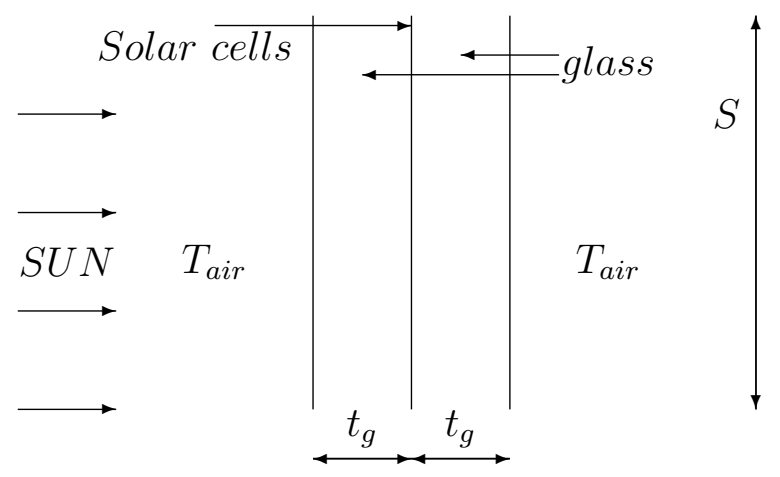

Figure 1: Cross sectional schematic view of a photovoltaic panel

Due to the relatively high thermal conductivity of silicon and the negligible thickness of the solar cells $(\approx 300 \mu \mathrm{m})$, the solar cell can be considered as an isothermal heat power source. The thermal power is given by the absorbed solar radiation which much be reduced by the amount converted to electricity.

The thermal resistance of the glass is given by:

$$
R_{t h, g}=\frac{1}{2} \frac{t_{g}}{k_{g} S}
$$

where $k_{g}$ is the thermal conductivity of glass, $S$ the area of the solar panel and $T_{g}$ the thickness of the glass. The factor $1 / 2$ is due to the fact that the same kind of glass was provided on both sides for the panels which have been investigated. Heat will be transferred to the ambient by convection and radiation. If $h$ denotes the total heat transfer coefficient, the related thermal resistance is given by:

$$
R_{t h, c}=\frac{1}{2} \frac{1}{h S}
$$

Using the following numerical values: $t_{g}=2 \mathrm{~mm}, k_{g}=0.8 \mathrm{~W} / \mathrm{mK}$, $S=0.1 \mathrm{~m}^{2}, h=10 \mathrm{~W} / \mathrm{m}^{2} \mathrm{~K}$ one gets: 


$$
R_{t h, g}=0.0125 \frac{K}{W} \quad \text { and } \quad R_{t h, c}=0.5 \frac{K}{W}
$$

The thermal resistance of the glass turns out to be much smaller, so that only the heat transfer to the ambient will be taken into account to set up a simple thermal model. The total thermal resistance turns out to be:

$$
R_{t h}=R_{t h, g}+R_{t h, c} \approx R_{t h, c}
$$

The thermal capacity of the glass is given by:

$$
C_{t h}=2 c_{v} t_{g} S
$$

where $c_{v}$ denotes the specific heat of glass per unit volume. The factor 2 is again due to the fact that two windows are used here. Using $c_{v}=$ $2.24 \mathrm{MJ} / \mathrm{m}^{3} \mathrm{~K}$ for glass, one gets:

$$
C_{t h}=896 \mathrm{~J} / \mathrm{K}
$$

The thermal capacity of the air boundary layer can be neglected due to the low specific heat of air being $1.7 \mathrm{~kJ} / \mathrm{m}^{3} \mathrm{~K}$. Using $R_{t h}$ and $C_{t h}$ one obtains the thermal equivalent network shown in Fig. 2.

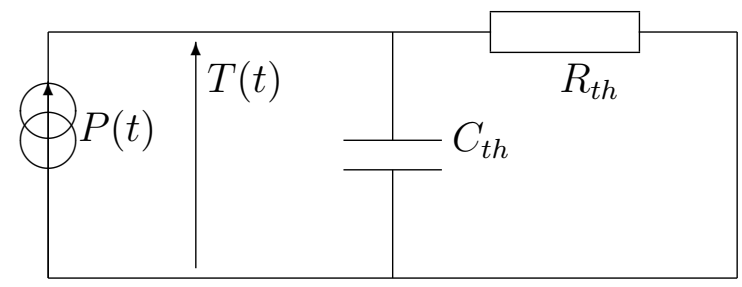

Figure 2: Thermal model for the solar panel

$P(t)$ is the heat produced by the cells and $T(t)$ the temperature rise of the cells above the ambient air $\left(T_{\text {air }}\right)$. This temperature satisfies the equation:

$$
C_{t h} \frac{d T}{d t}+\frac{T}{R_{t h}}=P(t)
$$

If a power step $P(t)=P_{0} u(t)$ is applied where $P_{0}$ is a constant and $u(t)$ the unit step function, the solution is found to be: 


$$
T(t)=R_{t h} P_{0}\left[1-e^{-t / \tau_{t h}}\right]
$$

where:

$$
\tau_{t h}=R_{t h} C_{t h}=\frac{c_{v} t_{g}}{h}
$$

is the thermal time constant. For the numerical values (3) and (6)one gets $\tau_{t h}=448 \mathrm{~s}$.

\section{Experimental measurements}

The theory outlined in the previous section can be extended to:

$$
T(t)=P_{0} \sum_{i=1}^{n} R_{t h, i}\left[1-e^{-t / \tau_{t h, i}}\right]
$$

or more generally, with a continuous distribution of time constants:

$$
T(t)=P_{0} \int_{0}^{\infty} \varphi(\tau)\left[1-e^{-t / \tau}\right] d \tau
$$

An ingenious technique to determine the thermal time constant distribution $\varphi(\tau)$ from the unit power step response $T(t)$ has been developed by V. Székely $[6,7,8]$. This method will be used in this contribution to determine $\varphi(\tau)$ from the experimentally measured $T(t)$. The same technique has also been used intensively for the thermal characterization of semiconductor components and packages $[9,10,11,12,13,14,15,16]$.

It should be noted here that the same technique can be used with a cooling curve too. If the device has been heated with a constant power for a sufficient long time (till steady state has been obtained), the cooling curve can also be used because it is nothing else than the complement of the heating curve (10) or (11).

During the experiment, the solar panel was put in a dark environment to prevent any photovoltaic output. A constant forward current was fed in order to heat the solar cells up to a steady state temperature. At time $t=0$ the heating was switched off and a small sensing current was applied instead. From the voltage drop across the panel, its temperature rise could be monitored continuously. For these experiments the T3ster equipment from MICRED company was used. 


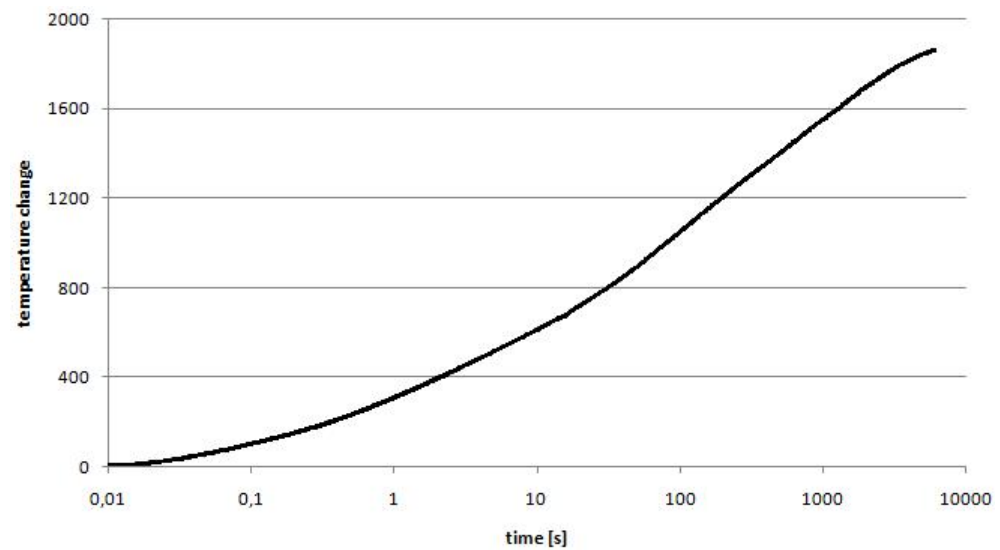

Figure 3: Experimentally recorded transient temperature of the solar cells

Fig. 3 shows a typical transient curve for a vertically placed solar panel. Similar curves have been obtained for inclinations of $0^{\circ}, 30^{\circ}, 45^{\circ}$ and $60^{\circ}$. The most remarkable result is that even after a few hours no steady state has been reached.

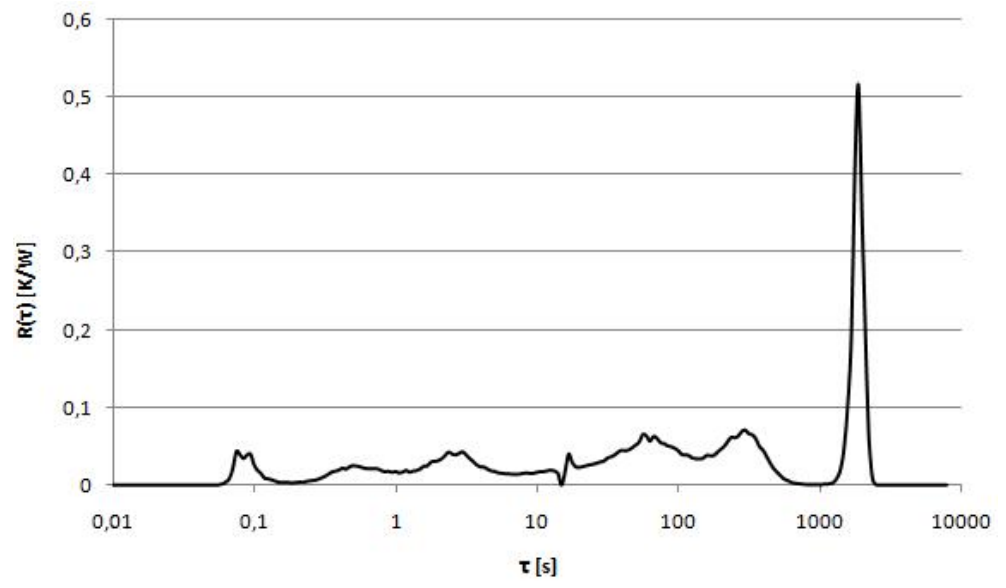

Figure 4: Time constant distribution spectrum of a solar panel

Fig. 4 displays the thermal time constant spectra calculated from the transient temperature curve of Fig. 3. A large peak is observed around 2 hours which corresponds with the transient result of Fig. 3. 


\section{Discussion}

The most remarkable result of Fig. 3 are the enormous thermal time constants of more than one hour. Even after 4 hours of measurements steady state has not been reached yet. Using the simple thermal model outlined above, one cannot imagine which heat transfer mechanism could be responsible for it. As similar results have been found for solar panels with different inclinations, an explanation has to be found in the special structure of the solar cells and more specifically the finger top contact has to be analyzed more closely.

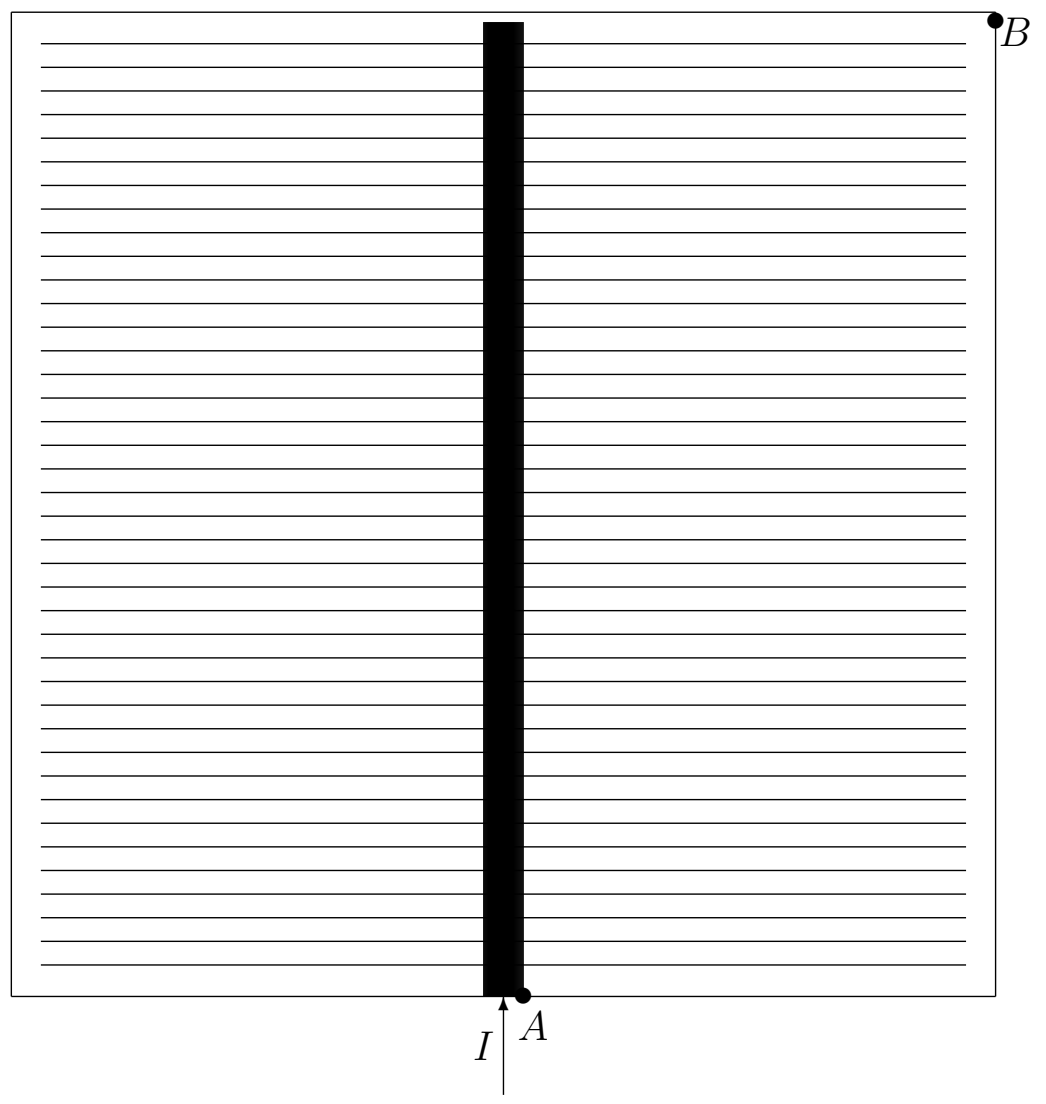

Figure 5: Top finger contact layout of a single solar cell

A layout of the finger top contact is shown in fig. 5. Each cell has an area of $10 \times 10 \mathrm{~cm}^{2}$. The distance between two parallel fingers is about $2.4 \mathrm{~mm}$. 
It should be noted that the series resistance of this kind of contact is not negligible. As a consequence the top surface of the solar cell may not be considered as an equipotential conductor. Not only the potential drop across the metallic contacts but also the potential drop between two parallel contact strips has to be taken into account.

If a constant current $I$ is supplied to the cell during the warming up phase, the potential in the point $A$ will be higher than in $B$. The amount of heat produced by the constant current $I$ will not be uniform over the entire cell, with a maximum in $A$ and a minimum in $B$. The solar cell being non uniformly heated at the start $t=0$ when the temperature recording starts, one has to consider the lateral diffusion of heat inside the polycrystalline silicon and the glass packaging as well.

Transient heat diffusion in a material with thermal conductivity $k$ and a specific heat $c_{v}$ per unit volume is described by the equation:

$$
k\left[\frac{\partial^{2} T}{\partial x^{2}}+\frac{\partial^{2} T}{\partial y^{2}}+\frac{\partial^{2} T}{\partial z^{2}}\right]=c_{v} \frac{\partial T}{\partial t}
$$

Normalizing all distances to a typical dimension $a$, one gets:

$$
\frac{\partial^{2} T}{\partial(x / a)^{2}}+\frac{\partial^{2} T}{\partial(y / a)^{2}}+\frac{\partial^{2} T}{\partial(z / a)^{2}}=\frac{\partial T}{\partial\left(t k / c_{v} a^{2}\right)}
$$

In other words, when all dimensions are normalized to a distance $a$, the time has to be normalized to:

$$
\tau=c_{v} a^{2} / k
$$

This value can be considered as a typical time the temperature needs to rise in a point at a distance $a$ from the heat source. For pure monocrystalline silicon one has $k=160 \mathrm{~W} / \mathrm{mK}$ and $c_{v}=1.6 \mathrm{MJ} / \mathrm{m}^{3} \mathrm{~K}$. Taking $a=10 \mathrm{~cm}$ as a typical dimension of the cell shown in fig. 5 one gets $\tau=100 \mathrm{~s}$. Taking into account the fact that the cells are made from polycrystalline silicon , a lower thermal conductivity can be expected and a higher time constant. Even when $k=40 \mathrm{~W} / \mathrm{mK}$ is used, the time constant is then $\tau=400 \mathrm{~s}$, still insufficient to explain the experimental measurement of Fig. 3.

A second cause of non uniform heating is the spreading between the individual solar cell $I-V$ characteristics. Each solar panel is made from 10 solar cells connected electrically in series (Fig. 6). Due to the non identical $I V$ characteristics, different voltages will be built up across each solar cell and consequently different power dissipations. At the beginning of the transient 


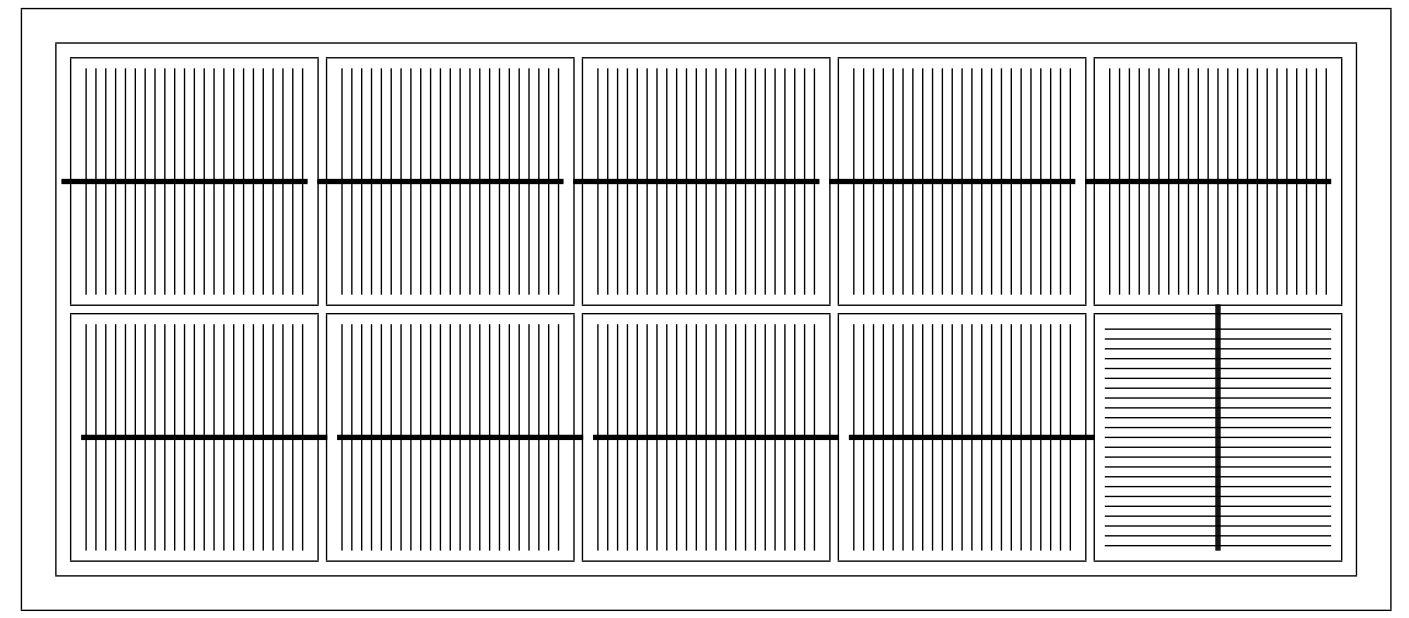

Figure 6: Layout of the solar cells in one solar panel

temperature measurements, one is dealing with a non uniform temperature distribution over the solar panel. During the measurement heat will diffuse from "warmer" to "colder" cells through the glass package in the lateral direction. Using $k=0.8 \mathrm{~W} / \mathrm{mK}$ for glass and $k=40 \mathrm{~W} / \mathrm{mK}$ for polycrystalline silicon, the average thermal conductivity in the lateral direction is given by:

$$
k_{\text {ave }}=\frac{40 T_{S i}+0.8 t_{g}}{t_{S i}+t_{g}}=3.5
$$

where $t_{S i}=0.3 \mathrm{~mm}$ and $t_{g}=4 \mathrm{~mm}$ have been used. For a typical distance $a=1 \mathrm{~cm}$ one obtains $\tau=64 \mathrm{~s}$ whereas for $a=10 \mathrm{~cm}$ a value $\tau=6400 s$ or almost two hours is found. This value also explain the long time constant observed in Fig. 3.

The same solar panel has also been measured experimentally, but the initial heating was done by solar irradiation, in order to guarantee a more uniform heating. The subsequent cooling curve could be fitted very well to an exponential decaying function with a time constant of 8 minutes or $480 \mathrm{~s}$ which agrees very well with the theoretical result (9) yielding $448 \mathrm{~s}$.

Fig. 4 also shows clearly a peak around $100 \mathrm{~ms}$. This peak can be easily explained by the heat diffusion through the thin layers of epoxy glue used to fix the solar cells between the two glass plates. Epoxy has a rather small thermal conductivity of only $k_{\text {epox }}=0.2 \mathrm{~W} / \mathrm{mK}$. The thermal capacity is $c_{\text {epox }}=2 \mathrm{MJ} / \mathrm{m}^{3} \mathrm{~K}$. Assuming a thickness $t_{\text {epox }}=50 \mu \mathrm{m}$ one gets for the 
related time constant:

$$
\tau_{\text {epox }}=\frac{t_{\text {epox }}^{2} c_{\text {epox }}}{k_{\text {epox }}}=100 \mathrm{~ms}
$$

which agrees with the experimental observation. A similar calculation for the polycrystalline solar cells $\left(k_{S i}=160 \mathrm{~W} / \mathrm{mK}, c_{S i}=1.6 \mathrm{MJ} / \mathrm{m}^{3} \mathrm{~K}\right.$ and $t_{S i}=300 \mu \mathrm{m}$ gives a thermal time constant around $100 \mu \mathrm{s}$, which could not be detected during the experiments. The same formula applied for the glass $\left(k_{g}=0.8 \mathrm{~W} / \mathrm{mK}, c_{S i}=2.24 \mathrm{MJ} / \mathrm{m}^{3} \mathrm{~K}\right.$ and $\left.t_{g}=2 \mathrm{~mm}\right)$ provides a thermal time constant of $10 \mathrm{~s}$ which is not visible in Fig. 4 because the glass is also convectively cooled which has not been taken into account in (16).

\section{Conclusion}

It has been pointed out that a knowledge of the thermal impedance of a photovoltaic panel provides useful information if used under fluctuating sunshine. In order to measure the thermal impedance classical method used for semiconductor devices should not be used.

Due to the large dimensions of a solar panel as compared to other electronic devices, the electric heating does not guarantee a uniform steady state temperature due to lateral heat diffusion. Heating with a uniform light source solves this problem.

[1] R. Dogniaux, M. Lemoine, R. Sneyers, Année - type moyenne pour le traitement de problèmes de captation d'énergie solaire, Belgian Royal Meteorology Institute, Brussels, 1978.

[2] W. Marańda, G. De Mey, A. De Vos, Optimization of the master-slave inverter system for grid-connected photovoltaic plants, Energy Conversion and Management 39 (12) (1998) 1239-1246.

[3] W. Marańda, D. Makowski, 1-kwp photovoltaic system at the technical university of łódź, Opto-electronics Review 12 (1) (2004) 75-77.

[4] G. De Mey, H. Simoens, Coupling between a photovoltaic panel and the power grid with battery storage, Electric Power Systems Research 4 (4) (1981) 283-288. 
[5] G. De Mey, H. Simoens, A. de Vos, Analysis of photovoltaic systems including a battery storage, International Journal of Electronics 51 (3) (1981) 215-220.

[6] V. Székely, T. Van Bien, Fine structure of heat flow path in semiconductor devices: A measurement and identification method, Solid State Electronics 31 (9) (1988) 1363-1368.

[7] V. Székely, Thermal monitoring of microelectronic structures, Microelectronics Journal 25 (3) (1994) 157-170.

[8] V. Székely, Identification of rc networks by deconvolution: Chances and limits, IEEE Transactions on Circuits and Systems I: Fundamental Theory and Applications 45 (3) (1998) 244-258.

[9] B. Vermeersch, G. De Mey, Thermal impedance plots of micro-scaled devices, Microelectronics Reliability 46 (1) (2006) 174-177.

[10] P. Kawka, G. De Mey, B. Vermeersch, Thermal characterization of electronic packages using the nyquist plot of the thermal impedance, IEEE Transactions on Components and Packaging Technologies 30 (4) (2007) 660-665.

[11] B. Vermeersch, G. De Mey, Influence of substrate thickness on thermal impedance of microelectronic structures, Microelectronics Reliability $47(2-3)(2007)$ 437-443.

[12] G. De Mey, B. Vermeersch, J. Banaszczyk, T. Swiatczak, B. Wiecek, M. Janicki, A. Napieralski, Thermal impedances of thin plates, International Journal of Heat and Mass Transfer 50 (21-22) (2007) 4457-4460.

[13] B. Vermeersch, G. De Mey, Influence of thermal contact resistance on thermal impedance of microelectronic structures, Microelectronics Reliability 47 (8) (2007) 1233-1238.

[14] M. Janicki, J. Banaszczyk, G. De Mey, M. Kaminski, B. Vermeersch, A. Napieralski, Dynamic thermal modelling of a power integrated circuit with the application of structure functions, Microelectronics Journal 40 (7) (2009) 1135-1140. 
[15] G. De Mey, J. Pilarski, M. Wójcik, M. Lasota, J. Banaszczyk, B. Vermeersch, A. Napieralski, Influence of interface materials on the thermal impedance of electronic packages, International Communications in Heat and Mass Transfer 36 (3) (2009) 210-212.

[16] G. De Mey, M. Wójcik, J. Pilarski, M. Lasota, J. Banaszczyk, B. Vermeersch, A. Napieralski, M. D. Paepe, Chimney effect on natural convection cooling of a transistor mounted on a cooling fin, Journal of Electronic Packaging, Transactions of the ASME 131 (1) (2009) 01450110145013. 